\title{
PROPERTIES, PHARMACEUTICAL APPLICATION AND VARIOUS TECHNIQUE OF CHEMICAL MODIFICATION IN NATIVE STARCH: A DESCRIPTIVE REVIEW
}

\author{
BISHAL JYOTI BORDOLOI*, BHUPEN KALITA, DIBYENDU SHIL \\ Girijananda Chowdhury Institute of Pharmaceutical Science (GIPS), Hatkhowapara, Azara, Guwahati 781017 Assam \\ Email: bishalkashyp17@gmail.com
}

Received: 18 Apr 2019, Revised and Accepted: 26 Jun 2019

\begin{abstract}
Starch is one important natural polymer that finds application in the formulation of dosage forms as the binder, disintegrates, diluents, gelling agent etc. Starch is drawing the attention in drug delivery as it is cheap, non-toxic, renewable, biodegradable and compatible with many other materials for industrial application. Starch has vital intrinsic properties that have made its pharmaceutical applications possible. It has also been used for a wide range of particular drug delivery applications, such as the delivery of challenging molecules and targeting to specific sites in the body. Starches are integrally unsuitable for most applications such as loss of viscosity and thickening power upon cooking and storage, retrogradation characteristics and absence of certain groups responsible for a particular function etc. So, in order to reduce its limitations and improve its applications, modification of starch is necessary. It can be modified by several ways like chemical modification, physical modification and genetic modification but the most important one is the chemical modification. This review summarizes the properties and application of native starchin conventional drug delivery systems within a world of dynamic drug production technology. It also describes the chemical modification like crosslinking, esterification, etherification and dual modification of starch.
\end{abstract}

Keywords: Starch, Chemical modification, Physiochemical properties, Cross-linking

(C) 2019 The Authors. Published by Innovare Academic Sciences Pvt Ltd. This is an open access article under the CC BY license (http://creativecommons.org/licenses/by/4.0/) DOI: http://dx.doi.org/10.22159/ijcpr.2019v11i4.34950

\section{INTRODUCTION [1-4]}

Starch is one of the most easily and widely available natural polymers. Starch can be obtained from various fruits and vegetables such as papaya, banana, corn, potato, rice, sago etc. The most common use of starch in our daily life is as a food ingredient. It is the most common source of glucose in the body. Starch has very low cost and significant properties which are being used or can be used for benefits of the human being. Starch is the principal form of the carbohydrate reserve in green plants and is formed in the cytoplasm of the plant cells in which they occur. Starch in its native form exists as a semi-crystalline molecule called grains or granules. The shape, size, and structure of the granules from each plant are different and are indeed characteristic of that botanical source. Native starch is starch isolated from its botanical source with minimal treatment such that the intrinsic physicochemical properties are maintained after processing. When kept dry, starch is stable in storage for a prolonged period. The attraction to the use of starch as a pharmaceutical excipient in various drug delivery technologies and formulations arise primarily due to their physicochemical and functional properties. Both native and modified starches are used as pharmaceutical excipients. Some essential attributes that make native starch attractive for use as a pharmaceutical excipient includes their white, soft, smooth dryness as well as gelling, and viscosity imparting properties. Also, when they are modified, new attributes are impacted which expand their functions and applications, making them more efficient in both conventional and novel drug delivery systems.

The techniques for starch modification have been broadly classified into four categories; physical, chemical, enzymatic and genetical modifications, that aim to produce various novel derivatives with improvised physiochemical properties along with useful structural attributes. There is a huge market for the many new functional and added value properties resulting from these modifications. A number of review articles on the subject of starch modification are available. However, in the last few years, there has been intense research by a various pharmaceutical professional to quest for an appropriate modification method that can yield a suitable candidate to be used as a pharmaceutical excipient in improvised drug delivery systems.

\section{Starch}

Synonyms: Amylum

Biological source

Starch consist of polysaccharide granules obtained from the grains of maize (zea mays linn); rice (oryza sativa linn.); or wheat (triticumaestivumlinn.); belonging to family graminae or from the tubers of potato (solanumtuberosum linn.), family solanaceae; seed of jackfruit (Artocarpusheterophyllus lam), family Moraceae.

\section{Description [5]}

Starch occurs as a fine powder and or angular, irregular masses readily reducible to powder. Colour of rice starch grains are white, while wheat is cream coloured and potatoes slightly yellowish, an odor of starch is odourless and the taste is mucilaginous. Size and shape of starch grains are very depending upon the types.

\section{Chemical constituents}

Starch contains chemically two different polysaccharides: amylase (Bamylose) and amylopectin ( $\alpha$-amylose), in the proportion of $1: 2$ amylose is water soluble and amylopectin is water insoluble, but swell in water and is responsible for the gelatinizing property of the starch.

Physiochemical properties of the starch

\section{Chemical structure [6]}

Pure starch is a white, tasteless and odourless powder that is insoluble in cold water or alcohol. It consists of two types of molecule:

Linear and helical amylase. Amylopectin.

Depending upon the source that is the type of plant, starch generally contains 20 to $25 \%$ amylase and 75 to $80 \%$ percent amylopectin by weigh 


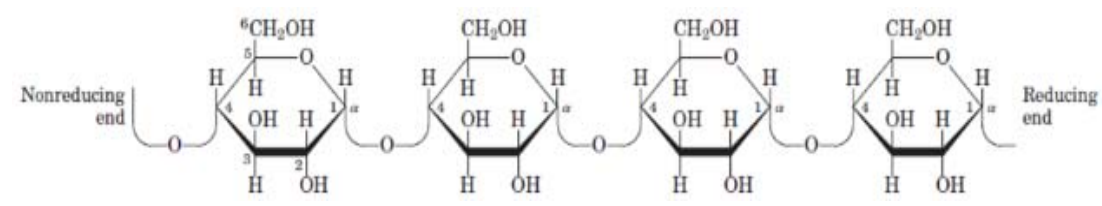

Fig. 1: Chemical structure of amylase



Fig. 2: Chemical structure of amylopectin

\section{Organoleptic properties [7]}

Typically, starch is a dry, soft powder that is odorless and bland in taste. The color varies depending on the botanical source, from white to slightly cream; however, starches of cereal origin may have colors that are shades of the seeds from which they are extracted. Sometimes the crude starch may have a slight odor that is characteristic of the botanical source. Though the intrinsic physicochemical properties of native starches are unaltered, many commercially available types are often subjected to certain physical treatment to enhance the elegance and modify certain physical properties such as whiteness and moisture content without altering the fundamental intrinsic properties.

\section{Morphology [7]}

Starch granule morphology is an important parameter used to identify and differentiate starch from different botanical origin. The general presentation, shape and sizes of starch granules from the different botanical origin are typical. Based on size description, starch grains can be classified into four categories: large $(>25 \mathrm{~mm})$, medium $(10-25 \mathrm{~mm})$, small $(5-10 \mathrm{~mm})$, and very small $(5 \mathrm{~mm})$. The morphology of many starch grains has been evaluated using the simple light microscope and the scanning electron microscope (SEM).

\section{Light scattering and iodine reaction [8]}

The appearance is such that under polarized light starch has a birefringence appearance. The amylose chain is coiled in the shape of a helix and is responsible for the characteristic purple-black color produced in the presence of iodine ions. The color reaction of iodine dissolved in potassium iodide solution to form the linear triiodide ion complex that enters the helical structure of the amylose moiety is an important identification test for starch. The purple-black color is formed when the iodine ions insert itself into the helical network of the amylose chain making it rigid. The change in color of the starch to either blue or purple will depend on the length of the amylose molecule. Amylopectin is a branching molecule which does not form a helical coil. Thus, the iodine is not able to bind to it.

\section{Crystalline properties $[9,10]$}

Native starch is described to be semi-crystalline or partly crystalline in nature with varying levels of crystallinity that is associated with the amylopectin and amylose content. The amylose and amylopectin polymers which make up the starch grain define the crystallinity of the unmodified starch. While the amylopectin represents predominantly the crystalline domain of native starch which is characterized by an ordered, tightly packed parallel glucan chains the amylose present the amorphous region. Starches from different botanical varieties have shown different crystalinity due to variation in their amylose and amylopectin content. Melting transitions are typically characteristic of the crystalline portion which corresponds to the amylopectin moiety.

\section{Solubility [11]}

Typically native starch is insoluble in cold water and most organic solvents including acetone, alcohols, and ether. It, however, becomes soluble in water when the dispersion is heated up to a certain critical temperature called the gelatinization temperature. Gelatinization is a fundamental property of starch which is characterized by changes in the physical and chemical properties. The process of gelatinization is characterized by enormous swelling, increased viscosity, translucency, solubility, and loss birefringence. These changes are often due to the breaking of the hydrogen bonds within the starch granules that allows water to enter the granules to make it to swell as the dispersion is heated. As the temperature increases, the viscosity of the dispersion also increases until a stable gel is formed. It is also important to note that as the temperature of the dispersion is increased stirring will further increase the viscosity of the dispersion. Gelling is characterized by high viscosity and a total destabilization of the crystalline structure of the granules followed by retrogradation which occurs upon cooling of the gel.

\section{Gelation [12]}

Starch gel is formed when starch paste in sufficiently highconcentration is cooled. Gelation is said to occur by the formation of a three-dimensional network in which swollen granules are bound. Amylose is considered to be primarily responsible for the formation of gels. The more the amylose-content, the stringier the gel while the more the amylopectin,the softer and translucent the gel. Among the official searches, potato starch has the highest amylopectin content which is responsible for its higher thickening potential as compared to corn starch which has lower amylopectin content and a stronger gel.

\section{Retrogradation $[6,13]$}

This is an important feature of starch gel which involves a molecular reorganization process characterized by loss of water and partial recovery of the crystalline structure due to the realignment of amylose and amylopectin. The firmness or rigidity of starch gel increases markedly with retrogradation. Retrogradation is induced by such factors as low temperature, high amylose content, and the presence of polar substances, such as salts. On the other hand substances such as surfactants, sugars, lipids, and hydrocolloidshinder retrogradation. Generally, the starches with 
higher amylose content will undergo retrogradation at a more rapid rate than those with higher amylopectin.

\section{Pharmaceutical applications of starch}

Disintegrating agent. Binder. Film-forming material. Microspheres. Colon targeting of drugs. Nanoparticle.

\section{Disintegrating agent [13-18]}

Starch is indeed the most commonly used disintegrating agent in the formulation of tablets. Tablet disintegrants are the hydrophilic substances which swell by absorbing the water in the gastrointestinal tract. This water absorption leads to swelling and hence disintegration of the tablet into smaller fragments. Native starch is a classical disintegrant and remain the popular disintegrant used in many conventional tablets and capsules of generic and branded drug products. Maize and potato starches are the most commonly used starch types for this purpose. However, empirical evaluations of starches from other botanical sources has also shown potential as an effective disintegrant in oral rapid release tablets and capsules. The various excipients used as a disintegrant in conventional rapid release tablets and capsules have different mechanisms of action. However, the mechanisms established for the disintegration ability of native starch are wicking and restoration of deformed starch particles on contact with aqueous fluid. When starch compact wick up water, the hydrogen bonds formed during compaction become disrupted, a phenomena which is further enhanced by the elastic deformation property of starch and the compact loosen-up and disintegrate. The optimal conditions required for the activity of starch as a disintegrant are usually: sufficient quantity of starch in the agglomerates, low compression pressure, and the presence of water. Generally, a good disintegrant must be effective at low concentrations to avoid or reduce its influence on the other properties of the tablet such as hardness, friability, and compaction ability. The properties of the API and other adjuncts are also influential in the efficiency of starch as a disintegrant. As a disintegrant, native starch is employed within the range of $3-25 \% \mathrm{w} / \mathrm{w}$ of the granules' or tablets' weight, a typical concentration is $15 \% \mathrm{w} / \mathrm{w}$. During granule formulations, optimal disintegrant activity is obtained when half of the starch is included in the granulation mixture as endo-disintegrant while the other half is incorporated directly to the dried granules as exodisintegran. The optimum amount of starch that will be used is often determined by optimization studies. When the amount of starch is below the optimum concentration there will be insufficient channels for capillary action thus, ingress of water will be hampered. Also, when the amount of starch is in excess, soft tablets with high friability and tendency to cap will result. These constitute a major drawback in the use of native starch as a disintegrant, thus the drive to develop motifs with enhanced disintegration and compaction abilities.

\section{Binder}

The most common binder used in tablet granulation is starch paste. The gelling property of starch is responsible for the binding property. Mainly $2-10 \% \mathrm{w} / \mathrm{v}$ solution of starch is used as a tablet binder. Amylose part of the starch is responsible for its gelling as well as binding property. Freshly made starch mucilage prepared to gel by heat treatment of the starch dispersion in water has been used extensively as the binder in tablets and capsules production using the wet granulation technique. The starch mucilage is incorporated as a gel and functions as a glue to provide the necessary binding force that holds the powder particles together to form the required agglomerates. This also serves to ensure uniform distribution and controllable release of the active pharmaceutical ingredient (API). When the agglomerates are compressed under optimal load, stable robust tablets are formed. Another important attribute of starch, when used as the binder, is its compatibility with other adjuncts and APIs used in many conventional and novel formulations. The powder mix often comprise of the APIs and other excipients which is granulated to produce a uniform distribution of the API and controllable drug release. When the starch gel is used as a binder, the granules are produced by wet granulation process usually by massing the powder mix and the starch gel to form a wet mass which is then screened through sieves of appropriate mesh size (1000-1700 $\mathrm{mm}$ ), depending on the range of the granule size desired. The agglomerates are then dried in a hot air oven at a temperature of between 40 and $60{ }^{\circ} \mathrm{C}$ for $1-6 \mathrm{~h}$ depending on the nature of the API or other excipients or desired formulation type. Native starch gel is incorporated at concentrations of $3-20 \% \mathrm{w} / \mathrm{w}$ of the granule or tablet weight. Typical concentration is usually $5-10 \%$ $\mathrm{w} / \mathrm{w}$, depending on the starch type. The optimal quantity of the gel required to produce robust granules or tablets is predetermined by optimization studies using any or combinations of such parameters as granule flow, tablet friability and hardness, disintegration time, and dissolution rate. When used appropriately within the precise optimal concentrations for granule formulation it will add sufficient cohesion to the powder mix to produce granules of desirable and optimal physio-mechanical properties that can be packed into sachets, capsule shells or compressed into tablets. Among the official starches, corn starch is the most frequently used in conventional granulation and tablet production technology, however various studies indicate that other starches from non-conventional sources have also shown potential as binder and so could be substituted for corn starch.

\section{Film forming polymer [19]}

Starch is chemically composed of two parts,amylose and amylopectin. The amylose content of starch is responsible for its film-forming property. Starch is a natural polymer and biocompatible and biodegradable. These properties of starch make it suitable for human use. The starch films can be used for drug delivery also. The film formulated with sago starch and lemongrass oil has been evaluated for its antimicrobial activity. Starch also has mucoadhesion property so it can also be used for sustaining drug release as mucoadhesive drug delivery systems. To improve the film-forming property of native starch it can be combined with various other polymers such as PVP, chitosan, sodium alginate etc.

\section{Microparticular drug delivery [20]}

Microspheres provide various advantages as drug delivery system. Starch has been used as coating polymer for delivery of various drugs through microspheres. The effect of cross-linking conditions on the properties of starch microspheres. The particle size increased with increasing the cross-linking time and increasing the drug loading. The swelling ratio of the particles was a function of crosslinker type but not the concentration or time of cross-linking. Drug release from starch microspheres was measured in phosphate buffer and also in phosphate buffer containing a-amylase. Results showed that microspheres cross-linked with epichlorhydrine released all their drug content in the first $30 \mathrm{~min}$. However, cross-linking of the starch microspheres with glutaraldehyde or formaldehyde decreased drug release rate.

\section{Colon targeting [21]}

A novel tablet of protein drug matrix for colon targeting using resistant starch as a carrier prepared by pre-gelatinization and cross-linking of starch. Cross-linked pregelatinized maize starches by double modification of pregelatinization and cross-linked with POCl3. They used bovine serum albumin as a model drug.

The content of resistant starch and swelling property of maize starch were increased by pre-gelatinization and cross-linking, which retarded its enzymatic degradation. The results indicate that the resistant starch carrier prepared by pregelatinization and crosslinking can be used for a potential drug delivery carrier for colontargeting drug matrix delivery system.

\section{Starch-based nanoparticle [22]}

Starch-based nanoparticles have been used for the transdermal delivery of the drugs like flufenamic acid, testosterone and caffeine. The skin permeation data for the three drugs suggest that starch nanoparticles have the potential for transdermal drug delivery applications. Encapsulation and release properties of these nanoparticles were studied, showing high encapsulation efficiency for three tested drugs (flufenamic acid, testosterone and caffeine); in addition, a close to linear release profile was observed for hydrophobic drugs with a null initial burst effect. 
Chemical modifications of starch and application of its derivatives [23-24]

Chemical modification involves the introduction of functional groups into the starch molecule, resulting in markedly altered physicochemical properties. Such modification of native granular starches profoundly alters their gelatinization, pasting and retrogradation behavior. The chemical and functional properties achieved when modifying starch by chemical substitution depend, inter alia, on starch source, reaction conditions (reactant concentration, reaction time, $\mathrm{pH}$ and the presence of catalyst), type of substituent, extent of substitution (degree of substitution, DS1; or molar substitution, MS2), and the distribution of the substituents in the starch molecule. Modification is generally achieved through derivatization such as etherification, esterification, cross-linking and grafting of starch; decomposition (acid or enzymatic hydrolysis and oxidization of starch). These techniques are however limited due to issues concerning costumer's nd the environment. These techniques include:

\section{Etherification and esterification [25-29]}

This method primarily includes substitution of hydrophilic hydroxyl groups of starch by various hydrophobic functional groups resulting into hyproxypropylation, carboxymethylation, acetylation, succinylation, etc. The carboxymethyl substitution of starch hydroxyl groupsgives rise to derivatives that are cold water-soluble. Toprevent starch gelatinization, the reaction has to be carriedout in an organic medium. Carboxymethyl starch, under thename sodium starch glycolate, is used in the pharmaceuticalindustry as a disintegrant and as a sizing and printing agent inthe textile industry. Highly substituted derivatives arepossible.

In acetylation, hydrophilic hydroxyl groups are substituted with hydrophobic acetyl groups. Acetylation makes starchmore hydrophobic and prevents the formation of hydrogenbonding between hydroxyl groups and water molecules. Since the tendency of an aqueous starch dispersion starches show resistance to high temperature,low $\mathrm{pH}$, high shear, and leads to increased stability of theswollen starch granule. They improve viscosity and textural properties of the starch. As a thickener and stabilizer, starch phosphate diesters are superior to unmodified starches. They also provide resistance to gelling and retrogradation, and donot synerese on storage.

Derivatization of starch with an ionic substituent group such as succinate at low degree of substitution (DS) converts it into a polyelectrolyte, which makes it acquire typical properties of a polyelectrolyte like increased hydrophiliccharacter and increased solution viscosity. This modification is also known to weaken the internal bonding that holds the granules together. In to increase in viscosity on cooling and finally to gel is related to the association of amylose molecules, a treatment such as acetylation which retards or eliminates this crystallization or retrogradation will effect stabilization of the starch sol. Acetylation also prevents or minimizes association of amylopectin outer branches. This is of practical value in many industrial and food applications because such associations can cause cloudiness and syneresis in aqueous dispersions of starches. Hydroxypropylated starches are generally prepared by etherification of native starch with propylene oxide in the presence of an alkaline catalyst. The hydroxypropyl groups introduced into the starch chains are capable of disrupting the inter-and intramolecular hydrogen bonds, thereby weakening the granular structure of starch, leading to an increase in motional freedom of starch chains in amorphous regions. Hydroxyethyl starch, prepared by reaction withethylene oxide, was of considerable biomedical interest as ablood plasma expander and also as a cryoprotective agent forerythrocytes. Starch phosphorylation is the earliest method of starchmodification. The reaction gives rise to either monostarchphosphate or distarch phosphate (cross-linked derivative),depending upon the reactants and subsequent reactionconditions. Monoesters, rather than diesters, are producedwith a higher level of phosphate substitution on starch. Theintroduction of phosphate substitution on amylose or outerbranches of amylopectin prevents linearity of molecularchains due to steric hindrance. Thus, a situation is achievedwhere individual chain segments can no longer approacheach other closely enough to establish intermolecular orintramolecular association; they, therefore, lead to a betterpaste clarity. The phosphate diester starches have thephosphate esterified with two hydroxyl groups, very oftenfrom two neighboring starch molecules. This leads to theformation of a covalent bridge or cross-linking. Phosphatecrosslinked addition starch succinate offers very desirable properties such as low-temperature stability, high-thickening power, and lowgelatinization temperature, clarity of cooked food, good film-forming properties and reduced tendency to retrograde. High cationic properties have been observed for starch citrates. The reaction is facilitated thermochemically by the dry heating $(\sim 120 \mathrm{C}$ for $24 \mathrm{~h})$ of citric acid to an anhydride, which reacts with starch to form an addict, followed by further reaction to yield cross-linked starch citrate. The reaction can be controlled to maximize reaction efficiency, minimize cross-linking, and maximize carboxyl content. They are used to remove toxic, heavy metal ions in water purification (industrial waste water) as biodegradable ionexchange materials against the petrochemically derived ionexchange resins

\section{Cross-linked starch [30-36]}

Cross linking is a chemical modification method during which the native starches are chemically modified using different cross linking agents like sodium trimetaphosphate (STMP), sodium tripolyphosphate (STPP), epichlorohydrin (ECH) and phosphoryl chloride (POCl3). This technique is believed to play an important role in improving the functional properties, the freeze thaw and the cold storage stabilities of the starch molecule. STMP, STPP, POCl3 and a mixture of adipic acid and acetic anhydride can be used as crosslinking agents for food use. Among these reagents, the STMP, which is one of the most important food additives with low toxicity, is an efficient cross-linking agent. One type of cross linked starch is distarch phosphate whichis produced under alkaline conditions by reacting starch granules with STMP or POCl3. The process of cross linking is believed to add intra-and inter-molecular bond at various location in the starch molecule that resulted in the stability of the granuleby utilizing the chemical reagents that form ether or ester linkages between hydroxyl groups in the molecule of starch. It is reported that both amylopectin and amylose in starch granules can be cross linked.

The amylose molecules, however, has less of the tendency to be cross-linked. Likewise, other chemical modification methods, cross linking of starches is also affected by many factors like source of the starch, the reagent composition and concentration, $\mathrm{pH}$, extent of substitution, temperature and reaction time. The molecular structure of starch granules is different by using different types of cross linkingagents; therefore, the functional properties of treated starch are determined by the type of cross linking agent used. The solubility of the cross linked starches was decreased with an enhancement in the degree of cross linking when measured as a function of temperature. Cross linked starches more suitable for various industrial applications as exhibited by their stronger granular structure. The most interesting phenomenon is that the cross linked starches decompose promptly but the amount of residues are larger after the decomposition process as compared to the untreated starches. The thermal decomposition of cross linked starches is facilitated by the large number of phosphate group in the amorphous region as evident by the XRD spectra of cross linked sago starch showing more amorphous region with reduced diffraction intensity that might be resulted due to the replacement of some hydroxyl groups with phosphate groups during the process of cross linking. Starch paste from cross-linked starch is highly viscous, heavy bodied, shorter textured and less likely to breakdown during severe agitation, cooking and exposure to low $\mathrm{pH}$ conditions. These starches are thus suitable for thickening and stabilizing the food system, e. g. soups, gravies, sauces, baby foods, fruit fillings, pudding and deep fried foods.

\section{Acid treatment [37-38]}

In acid modification, the hydroxonium ion attacks the glycosidic oxygen atom and hydrolyses the glycosidic linkage. An acid acts on the surface of the starch granule firstbefore it gradually enters the inner region. Acid modification changes the physicochemical 
properties of starch without destroying its granule structure and the properties of acidthinned starches differ according to their origin. The gelatinization temperature and the breadth of the gelatinization endotherm have also been shown to increase on acid hydrolysis. The retrogradation rate of acid thinned starch increased as hydrolysis proceeded. The method for the manufacture of acid thinned starch entails treating concentrated starch slurry with mineral acid at temperatures below gelatinization temperature for specific period depending on the desired viscosity or degree of conversion. Effect of different acids $(\mathrm{HCl}, \mathrm{HNO} 3, \mathrm{H} 2 \mathrm{SO} 4$ and $\mathrm{H} 3 \mathrm{PO} 4)$ under similar conditions of treatment on molecular weight, alkali fluidity number, iodine binding capacity and intrinsic viscosity of various starches has also been studied.

\section{Oxidation [39]}

Oxidized starch is produced by reacting starch with a specified amount of oxidizing reagent under controlled temperature and $\mathrm{pH}$. Oxidation causes depolymerisation, which results in a lower dispersion viscosity and introducescarbonyl and carboxyl groups, which retard Recrystallization.

\section{Dual modification [40-42]}

These include methods that involve the chemical reaction in the presence of a specific physical environment or an enzymatic treatment that make serve to enhance the rate of derivatization or can enhance the degree of substitution in some instances.
Microwave-assisted esterification to produce starch maleate using the dry method had a reaction efficiency of up to $98 \%$ and a reaction time of less than $5 \mathrm{~min}$. This is thought to be an efficient method in esterifying starch. The high efficiency in producing succinylated cassava starch with microwave assistance was. This is a good method to decrease the use of chemicals to enhance production. Microwave and ultrasound irradiation was used for the Esterification of carboxymethyl cold-water-soluble potato starch with octenyl succinic anhydride. They were positively able to shorten the esterification time from a few hours to a few minutes. The derivatives displayed excellent emulsifying and surfactant performance properties. A combined methodof modification using crosslinking and phosphorylation on rice starch provided modified rice starch with good freezethawstability. Modified corn and mung bean starch was prepared bytreating native starch with a mixture of fungal $\alpha$-amylase andglucoamylase followed by hydroxypropylation withpropylene oxide. This partial enzymatic hydrolysis producesmodified starch that possesses significantly differentfunctional properties to that of hydroxypropyl starch preparedunder normal conditions. The use of fungal lipase to enhance the Esterification of cassava starch using recovered coconut oil gives thermoplastic starch which has got wide use in plastic industry, pharmaceutical industries, and in biomedical applications such as materials for bone fixation and replacements, carriers for controlled release of drugs and other bioactive agents. Unlike chemical esterification, enzymatic esterification is ecofriendly and avoids the use of nasty solvents.

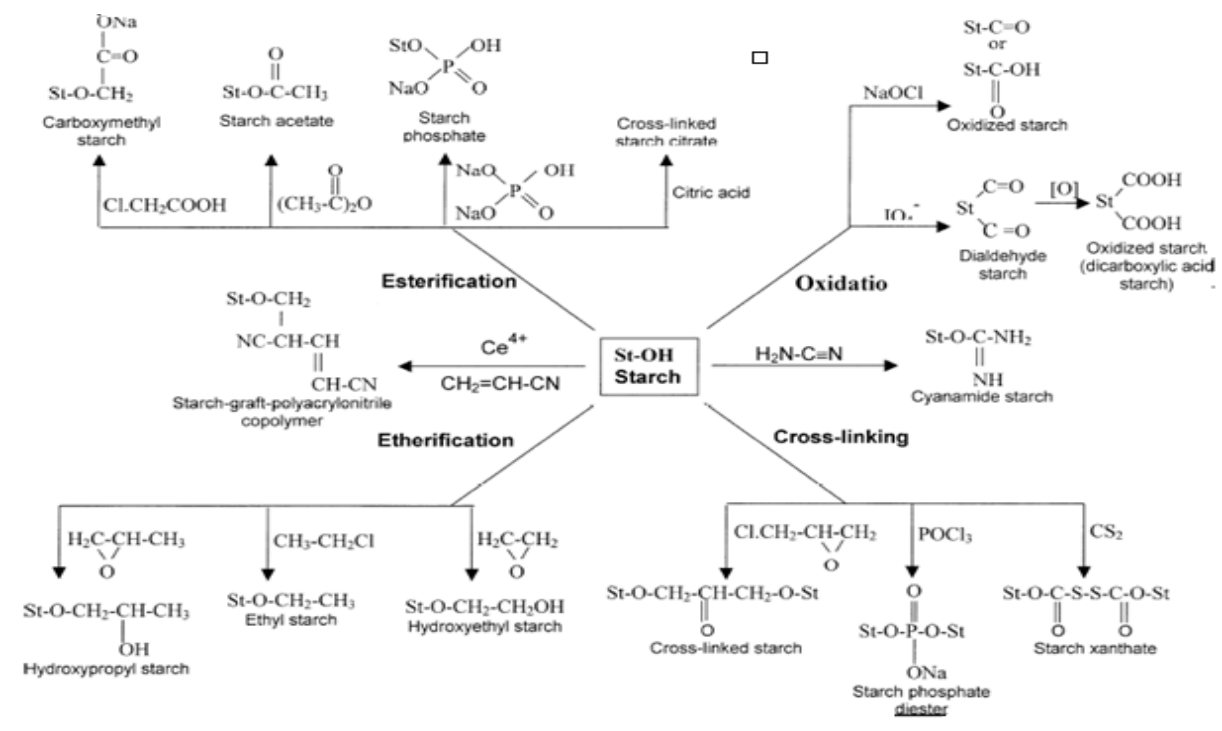

Fig. 3: Chemical modification technique [43]

\section{CONCLUSION}

Starch is very important excipient and it can fulfill various requirements for the delivery of the drug. Starch is widely used in the production of especially oral solid dosage form such as bulk granules, tablets and capsules as well as cosmetic and medicated powder. Native starch as conventional dosage form such as binder, disintegrate, diluent, glidant, lubricant. Starch is a natural biodegradable biopolymer of the plant origin which is modified by various methods to improve its useful properties and eradicate its negative impacts, which limit its use. Among various techniques used for the modification of starch, chemical modification is the most important and advance technique which mainly includes crosslinking, esterification, etherification and dual modification of starch.

\section{AUTHORS CONTRIBUTIONS}

All the author have contributed equally

\section{CONFLICT OF INTERESTS}

Declare none

\section{REFERENCES}

1. Philip FB, Mathew IA. Pharmaceutical application of native starch in conventional drug delivery. Starch/Starke 2016;68:1-10.

2. BeMiller JN. Starch modification: challenges and prospects. Starch/Starke, 1997;49:127-31.

3. Jobling S. Improving starch for food and industrial applications. Curr Opinion Plant Biol 2004; 7:210-8.

4. Tharanathan RN. Starch-value addition by modification. Crit Rev Food Sci Nutr 2005;45:371-84

5. Kokate CK, Purohit AP, Gokhle SB. A textbook of pharmacognosy. 48 th edition. Nirali Prakashan; 2011. p. 8.308.34 .

6. Adeleke OA. Comparative characterization of the physicochemical properties of some starch blends-Bombarra ground nuts and cassava starch versus cocoyam and wheat starches. Int J Curr Res Acad Rev 2014;2:317-29.

7. Manek RV, Kunle OO, Emeje MO, Builders PF. Physical, thermal and sorption profile of starch obtained from tacca leontopetaloides. Starch/Starke 2005;57:55-61. 
8. Knutson CA. Evaluation of variationsinamyloseiodineabsorbance spectra. Carbohydrate Polymer 1999;42:6572.

9. Oates CG. Towards an understanding of starch granulestructure and hydrolysis. Trends Food Sci Technol 1997;8:375-82.

10. Stasiak K, Molenda M, Opalinski I, Błaszczak W. Mechanical properties of native maize, wheat, and potatostarches. Czech J Food Sci 2013;31:347-54.

11. Shimelis E, Meaza M, Rakshit S. Physico-cemicalproperties, pasting behavior and functional characteristics offlours and starches from improved bean (Phaseolus vulgaris L.) varieties grown in East Africa. Agric Eng Int 2006;VIII:1-19.

12. Rindlav Westling A, Stading M, Hermansson A, Gatenholm P. Structure, mechanical and barrier properties ofamylose and amylopectin films. Carbohydrate Polymer 1998;36:217-24.

13. Sasaki T, Yasui T, Matsuki J. Effect of amylose content on gelatinization, retrogradation, and pasting properties of starches from waxy and non-waxy wheat and their seeds. Cereal Chem 2000;77:58-63.

14. Pilpel N, Otuyemi SO, Kurup TRR. Factors affecting the disintegration and dissolution of chloroquine phosphate/starch tablets. J Pharm Pharmacol 1978;30:214-9.

15. Akin-Ajani OD, Itiola OA, Odeku OA. Effects of plantain and corn starches on the mechanical and disintegration properties of paracetamol tablets. AAPS PharmSciTech 2005;6:E458-E463.

16. Adebayo AS, Itiola OA. Evaluation of breadfruit and cocoyam starches as exo-disintegrantsina paracetamol tablet formulation. Pharm Pharmacol Commun 1998;4:385-9.

17. Nattapulwat N, Purkkao N, Suwithayapanth O. Evaluation of native andcarboxymethyl yam (Dioscorea esculenta) starches as tablet disintegrants. Silpakorn Univercity Sci Technol J 2008:2:18-25.

18. Lowenthal W. Mechanism of action of tablet disintegrants. Pharm Acta Helv 1973;48:589-609.

19. Myllarinen P, Buleon A, Lahtinen R, Forssell P. The crystallinity of amylose and amylopectin films. Carbohydrate Polymers 2002;48:41-8.

20. Atypi F, Manoochehri S, Moghadam SH, Dinarvanl R. Cross-linked starch microsphere effect of cross linking condition on the microsphere characterstic. Arch Pharm Res 2006;29:1179-86.

21. Chen L, Li X, Pang Y, Li L, Zhang X, Yu L. Resistant starch as a carrier for oral colon-targeting drug matrix system. J Materials Sci 2007;18:2199-203.

22. Santander Ortega MJ, Stauner T, Loretz B, Ortega Vinuesa JL Nanoparticles made from novel starch derivatives for transdermal drug delivery. J Controlled Release 2010;141:8592.

23. Hirsch JB, Kokini JL. Understanding the mechanism of crosslinking agents (POCl3, STMP, and EPI) through swelling behavior and pasting properties of cross-linked waxy maize starches. Cereal Chem 2002;79:102-7.

24. Wang YJ, Wang L. Characterization of acetylated waxy maize starches prepared under catalysis by different alkali and alkaline-earth hydroxides. Starch/Starke 2002;54:25-30.

25. Bentacur AD, Chel GL, Canizares HE. Acetylation and characterisation of canavalia ensiformis starch. J Agric Food Chem 1997;45:378-82.
26. Greenwood CT, Muir DD, Whitcher HW. Hydroxyethyl starch as a cryoprotective agent for human red blood cells. The relation between the molecular properties and the cryoprotective effect. Carbohydrate Polymers 2004;55:23-6.

27. Sitohy MZ, Labib SM, Saadany SS, Ramadan MF. Optimizing the conditions for starch dry phosphorylation with sodium monoand dihydrogen orthophosphate under heat and vacuum. Staerke 2000;52:95-100.

28. Nabeshima EH, Grossmann MVE. Functional properties of pregelatinized and crosslinked cassava starch obtained by extrusion with trimetaphosphate. Carbohydrate Polymers 2001; 45:347-53

29. Bhandari PN, Singhal RS. Preparation of sugarcane bagasse hemicellulosic succinates using NBS as a catalyst. Carbohydrate Polymers 2002;47:277-83.

30. Chung HJ, Woo KS, Lim ST. Glass transition and enthalpy relaxation of cross linked corn starches. Carbohydrate Polymers 2004;55:9-15.

31. Gui Jie M, Peng W, Xiang Sheng M, Xing Z, Tong Z. Cross linking of corn starch with sodium trimetaphosphate in solid state by microwave irradiation. J Appl Polymer Sci 2006;102:5854-60.

32. Wurzburg OB. Nutritional aspects and safety of modified food starches. Nutr Rev 1986;44:74-9.

33. Rutenberg MW, Solarek D. Starch derivatives: production and uses. Academic Press: London; 1984. p. 312-88.

34. Jane J, Xu A, Radosavljevic M, Seib PA. Location of amylose in normal starch granules. Susceptibility of amylose and amylopectin to cross-linking reagents. Cereal Chem 1992;69:405-9.

35. Seker M, Hanna MA. Sodium hydroxide and trimetaphosphate levels affect properties of starch extrudates. Industrial Crop Products 2006;23:249-55.

36. Singh AV, Nath LK. Synthesis and evaluation of physicochemical properties of cross-linked sago starch. Int J Biol Macromol 2012;50:14-8.

37. Koo SH, Lee KY, Lee HG. Effect of cross linking on physicochemical and physiological properties of corn starch Food Hydrocolloids 2006;24:619-25.

38. Singh V, Ali SZ. Acid degradation of starch. The effect of acid and starch type. Carbohydrate Polymers 2000;41:191-5.

39. Kuakpetoon D, Wang YJ. Characterisation of different starchesoxidised by hypochlorite. Starch/Starke 2001;53:2118.

40. Jyothi AN, Rajasekharan KN, Moorthy SN, Sreekumar J. Microwave assisted synthesis and characterization of succinate derivatives ofcassava (Manihot esculenta Crantz) starch. Starch/Starke 2005;57:556-63.

41. Cizova A, Srokova I, Sasinkova V, Malovikova A, Ebringerova A Carboxymethyl starch octenylsuccinate: microwave and ultrasoundassistedsynthesis and properties. Starch/Starke 2008;60:389-97.

42. Karim AA, Sufha EH, Zaidul ISM. Dual modification of starch via partial enzymatic hydrolysis in the granular state and subsequenthydroxypropylation. J Agric Food Chem 2008;56:10901-10907.

43. Kavlani N, Sharma V, Singh L. Various technique for the modification of starch and the application of its derivatives. Int J Pharm 2012;3:25-31. 